\title{
The Benefits of Preparing Data for Sharing Even When You Don't
}

\author{
David N. Kennedy
}

Published online: 4 June 2012

(C) Springer Science+Business Media, LLC 2012

Many journal pages (in this and other journals) have been filled with discussions, techniques and admonitions to share data. ${ }^{1}$ While it is true that enhanced data sharing is a critical (and lagging) step in the quest for more open, reliable, and reproducible science, the battle to reduce the social and ethical barriers remains a topic of much discussion. ${ }^{2}$ While the social and ethical discussion wages on, the loss of data and the potential for new insights that can be derived from it mounts.

Through data sharing efforts that have been undertaken, we have learned that the process of sharing often uncovers errors in the descriptive details of data. When data sharing is prospective (relative to when the results of the data may be published), these errors can be due to accidental mistakes in record keeping, misalignment of data from multiples sources, etc. Retrospective (post-publication) data sharing can compound the potential local errors in the actual data descriptors with additional errors in identification of the final specific data that was utilized in any specific publication. With the passage of time, and the inevitable changes in hardware, software, personnel, etc., it becomes difficult to reconstruct exactly what the data was that was included in a particular past publication. Data quality, for both local and

\footnotetext{
${ }^{1}$ D. N. Kennedy, "Share and share alike," Neuroinformatics 1, 211 (2003); D. N. Kennedy, "Barriers to the socialization of information," Neuroinformatics 2, 367 (2004); D. Kennedy, "Where's the beef? Missing data in the information age," Neuroinformatics 4, 271 (2006).

2 J. B. Poline et al., "Data sharing in neuroimaging research," Front. Neuroinform 6, 9 (2012).
}

D. N. Kennedy $(\bowtie)$

Division of Neuroinformatics, Department of Psychiatry,

University of Massachusetts Medical School,

Worcester, USA

e-mail: David.Kennedy@umassmed.edu shared purposes, is enhanced through the use of high-quality enduring lab notebook and local data management systems. One of the 'benefits' of data sharing is the identification and correction of potential errors in the data descriptions relative to the published data. ${ }^{3}$

By extrapolation from the errors that have been detected in the limited existing data sharing efforts, one must assume that there are numerous errors that have occurred in the reporting of data description in the published literature. For the most part, however, the extent, nature and prevalence of these errors are unknown. While often they may be innocuous with respect to the scientific conclusions derived from the data, the impact of such errors place extra burden on the ability of studies to be replicated, and hamper data aggregation for meta analyses.

Therefore, one way to avoid accidental data description error is to undertake the process of preparing the raw data of a publication for data archive/data sharing at the time of publication. There is a societal benefit (improved accuracy of published data description) that is generated by the preparation for sharing process even if the data is never actually shared with any outside user. Conceptually, the preparation for sharing offers an additional cross-validation step between the published description and what has been prepared for archive. This is analogous to the rational for why 'double entry' is standard process in many critical data acquisition procedures. Even if compelling arguments can be made for why a specific dataset should not be publically shared at the time of publication, it is possible that future advances in the technology, societal pressures, or ethical considerations may provide a solution to make sharing of these data possible. As prospective data sharing is both less error prone in the first

\footnotetext{
See forum discussions at http://www.nitrc.org/projects/fcon_1000 and http://www.nitrc.org/projects/candi_share for examples.
} 
place, and offers superior data correction opportunity, the process of preparation data for sharing (i.e. data archival) should be a requirement of publication.

Indeed, many journals do have mandatory data sharing policies. ${ }^{4,5}$ For example, The Public Library of Science (PLOS) Medicine publication policy states "Publication is conditional upon the agreement of the authors to make freely available any materials and information described in their publication that may be reasonably requested by others for the purpose of academic, non-commercial research". 6 However, adherence to these policies seems to be rather inconsistent. ${ }^{7}$ The inconsistency of actual data sharing when data sharing is required but not prospectively engaged should motivate the journals to take a stronger prospective role in encouraging (dare I say enforcing) the data sharing mandates.

One can make a distinction between 'data archival' and 'data sharing'. Both are fundamentally the same data preparation process, with the difference being that data archival may not necessarily result in data that will actually be publically shared. Since the data preparation objectives are similar (how the reader understands the details of data acquisition is equivalent to how a data user will need to understand the details of the data collection for future reuse) it can be suggested that the scope of data features that must be described and validated, i.e. the minimal necessary meta- data, will be identical in these two situations. The necessary minimal metadata is, of course, highly domain-specific and application specific (minimal metadata for diffusion MRI in humans is quite distinct from minimal metadata for electron microscopy of rat neurons). ${ }^{8}$ But the objective is common, to promote data understanding and comparison (and reuse in actual data sharing applications). Since these same minimum criteria are equally critical for interpretation of published results, the authors, reviewers, editors and publishers have an obligation to assure that all essential data descriptors are being reported accurately.

The archiving of data at the time of publication should be mandated. The archiving of data needs to become culturally an expected and obvious step of the research process (like keeping records of your experiments, writing papers, producing professional figures, giving talks at conferences etc.) Doing good research already 'takes time', and accuracy and reproducibility are essential elements to good research. A positive consequence of mandated archival, however, is that in the future one of the big barriers to sharing would fall. Sharing will still be a decision based on other factors (academic competition, privacy, quality, etc.), but at least it wouldn't also take more time than 'usual' research. In the end, at least for data derived from human patients, our commitment as a research community is to generate an answer (or cure) as quickly as possible.

\footnotetext{
$\overline{{ }^{4} \mathrm{http}: / / w w w . n a t u r e . c o m / a u t h o r s / e d i t o r i a l \_p o l i c i e s / a v a i l a b i l i t y . h t m l ~}$

${ }^{5}$ www.sciencemag.org/about/authors/prep/gen_info.dtl\# datadepwww.sciencemag.org/about/authors/prep/gen_info.dtl\#datadep ${ }^{6} \mathrm{http} / / /$ journals.plos.org/plosmedicine/policies.php\#sharing

${ }^{7}$ Savage CJ, Vickers AJ (2009) Empirical Study of Data Sharing by Authors Publishing in PLoS Journals. PLoS ONE 4(9): e7078. doi:10.1371/journal.pone.0007078
}

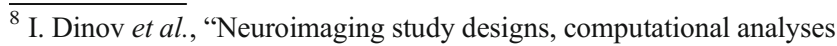
and data provenance using the LONI pipeline," PLoS One 5, e13070 (2010); G. Frishkoff et al., "Minimal Information for Neural Electromagnetic Ontologies (MINEMO): A standards-compliant method for analysis and integration of event-related potentials (ERP) data," Stand. Genomic Sci. 5, 211 (2011).
} 